\title{
Entwicklung und Ausprägung des Kinderfernsehens in Australien
}

von Ingrid Geretschlaeger

"Watching Telly" ist eine, wenn nicht die, Lieblingsbeschäftigung australischer Kinder. Im Alter zwischen vier und sieben Jahren sehen sie im Durchschnitt zwei bis fünf Stunden pro Tag, als Zwölfjährige zwischen drei und vier Stunden fern. 56\% der Zehn- bis Zwölfjährigen kommen auf mehr als drei Stunden täglich, 44\% der 14jährigen auf mehr als vier Stunden und 17\% gar auf mehr als fünf bis sieben Stunden täglich - mehr als sie jemals mit Schule oder Spiel verbringen ${ }^{1}$. Der Trend geht jedoch (erfreulicherweise) zu weniger Fernsehkonsum.

Bei solchen Fakten erstaunt es nicht, wenn sich Eltern, Lehrer und sonstige Verantwortliche Gedanken machen, wie man den eventuellen Schaden bei den Kindern verhindern kann bzw. wie das Angebot um speziell für Kinder gemachte Sendungen bereichert werden könnte. Als vorläufiger Endpunkt dieser Uberlegungen steht die Gründung der Australian Cbildren's Television Foundation, die Anfang 1982 ihre Tätigkeit aufnahm.

Der dritte Bericht (Mai 1982) des Komitees für das Kinderfernsehen ${ }^{2}$ und das Bekanntwerden dieser Stiftung für das Kinderfernsehen auch in Europa durch den Prix Jeunesse bieten einen guten Anlaß, um über die Situation des Fernsehprogrammangebots für Kinder in Australien zu berichten ${ }^{3}$

\section{Kinder und kommerzielles Fernseben}

Auch im kommerziellen Fernsehsystem Australiens ist für das Kinderfernsehen wenig Geld vorhanden. Gute Fernsehproduktionen sind teuer - gleich ob für Kinder oder Erwachsene $e^{4}$, aber Erwachsene versprechen über die Werbung wieder etwas einzubringen. Fernsehen ist ein Massenmedium, muß sich also prinzipiell verschiedenen Gruppen widmen, so viele wie möglich gleichzeitig ansprechen. Minderheiten (Kinder machen nur 23\% der Gesellschaft aus) sind nicht gefragt. Unter diesen Umständen sind Regelungen des Programmangebots für Kinder eine wesentliche Voraussetzung für das Zustandekommen von Kindersendungen überhaupt. Ohne sie gäbe es auch kein eigenes Kinderfernsehen ${ }^{5}$.

\section{Zur Geschichte der Reglementierungen}

Die allerersten Ansätze der Fernsehstationen, qualitativ hochwertiges Material oder zumindest für Kinder geeignetes Material auszustrahlen, lagen in den Versprechen der Fernseh-Anstalten, die bei Gewährung einer Lizenz gegeben wurden. Als jedoch der Fernsehalltag eingekehrt war, ließ man in der Routine die Kinder bald zu kurz kommen.

Dr. phil. Ingrid Geretschläger ist nach mehrjähriger Tätigkeit als Lehrerin und der Auswertung ihrer Erfahrungen in der Medienerziehung in einem Studium der Publizistikwissenschaft freie wissenschaftliche Mitarbeiterin in Salzburg. 
Familienprogramme wurden stillschweigend auch als Kinderprogramm deklariert. Es gab zwar seit 1956 ein Children's Television Advisory Committee, installiert vom Broadcasting Control Board (1971 wurden Richtlinien für Kinderfernsehen erarbeitet), aber bis 1975 tat sich nichts Wesentliches, und der Druck der Vereinigung der kommerziellen Fernsehstationen (F.A.C.T.S. - Federation of Australian Commercial Television Stations) auf den Vorsitzenden des Komitees gegen die Einführung von verbindlichen Regeln siegte vorerst.

Eine wesentliche Verbesserung der Situation begann erst, als 1978 in Adelaide in Südaustralien die ersten öffentlichen Lizenzverlängerungsverfahren begannen und die Offentlichkeit ein Forum für die Sorgen in bezug auch auf Kinder und Fernsehen bekam und diese Möglichkeit nutzen lernte.

Zur wachsenden Besorgnis in der Offentlichkeit hat wohl das zunehmende Bewußtsein für Kinder und das bevorstehende Jahr des Kindes beigetragen, sicher aber auch der Bericht über die Anhörung des Senate Standing Committee on Education and the Arts zum Problem des Einflusses des Fernsehens auf die Entwidklung und das Lernverhalten von Kindern ${ }^{8}$. Der Bericht umfaßt Ergebnisse von Untersuchungen zum Sehverhalten der Kinder, $\mathrm{zu}$ Wirkungen, eine Ubersicht über das Angebot und nicht zuletzt Vorschläge für die Verbesserung des Angebots sowie den Hinweis auf Medienerziehung und die Anregung zur Entwicklung eines Curriculums, gültig für alle australischen Schulen.

So wurde der Grundstein für eine (langjährige) Vorgangsweise gelegt, die sowohl die Schulung des Rezipienten als auch die Einsicht der Programmverantwortlichen anstrebt. Ende 1978 (der Australian Broadcasting Control Board war durch das Australian Broadcasting Tribunal ABT ersetzt worden) betraute das ABT ein Children's Program Committee - bestehend aus drei Mitgliedern der Industrie und vier aus der Offentlichkeit - mit der Aufgabe, Richtlinien für Kinderfernsehen zu erstellen und Klassifikationskriterien für die Beurteilung von Programmen, die in einer bestimmten Zeitspanne ausgestrahlt werden sollten, auszuarbeiten. Die Kommission selbst sollte jedoch keinerlei Autorität haben, sie sollte nur beratend wirken.

Vorschläge dieser Gruppe lagen noch im ersten Halbjahr 1979 vor, und mit 1. Juli 1979 traten u. a. folgende Erfordernisse für die kommerziellen Fernsehstationen in Kraft: 1 . sie haben $z$ wischen 16.00 und 17.00 Uhr (Montag bis Freitag) pro Woche nicht weniger als drei Stunden C-klassifiziertes Material (d. h. speziell für Kinder von sechs bis 13 Jahren produziertes Programm) auszustrahlen; 2. mindestens 30 Minuten des Programmangebots müssen für Vorschulkinder geeignet sein.

Schon bei Inkrafttreten der neuen Reglementierungen wurde angekündigt, daß sich die Forderungen erhöhen würden, sobald genug "gutes“ Material vorhanden sei. Und bereits ab 1. Juli 1980 waren dann fünf Stunden genehmigtes, C-klassifiziertes Material, als jeden Tag eine volle Stunde Kindersendungen zwischen vier und fünf Uhr nachmittags anzubieten ${ }^{7}$. Gleichzeitig wollte man jedoch nicht enge Richtlinien setzen, sondern kreative Bemühungen fördern.

Als Hilfe für die Produzenten von Kindersendungen wurden vom ABT inzwischen zwei Broschüren herausgegeben: „The Developing Child" ${ }^{8}$ beschreibt die Charakteristiken der verschiedenen Altersstufen und bringt Ideen für Sendungen für verschiedene Interessengruppen; die sehr unterhaltsam und ansprechend gestaltete Broschüre „Kid's TV. 433 Things to Do in a Rainy Studio ${ }^{\text {"9 }}$ ist ein nach Stichworten geordnetes Handbuch, das von der Liebe zu Kindern gekennzeichnet ist. 
Durch diese Maßnahmen sollten Kinder die Welt kennenlernen können, in der sie leben. Sie sollten lustige und traurige Geschichten geboten bekommen, die sie einbeziehen, die sie befähigen, in die Gesellschaft hineinzuwachsen. Man gesteht ihnen zu, dieselben Wünsche und Bedürfnisse zu haben wie die Erwachsenen und zwar nach menschlichen Situationen, komischen und tragischen, nach aufregenden Szenen und Action, nach Musik und Wettbewerb ${ }^{10}$.

\section{Wunsch und Wirklichkeit}

Bis 1978 waren $90 \%$ des Fernsehangebots zwischen 16.00 und 17.00 Uhr an die Familie gerichtet. Das Programm umfaßte Cartoons, Situationskomödien, Abenteuerserien, Quizsendungen usw., zum Großteil aus der Produktion vor 1970 (und den Vorstellungen der 50er und 60er Jahre) und zu 70\% amerikanischen Ursprungs, wobei die Sendungen des öfteren (manche bis zu zehnmal) wiederholt wurden. Kinderprogramm bzw. Programm zwischen 16.00 und 18.00 Uhr bedeutete bei den Fernsehstationen der großen Städte an Wochentagen zu zirka 60\% Zeichentrickfilme und Komödien, zu $11 \%$ Abenteuerserien, 9\% war der Beitrag zur Kinderquote, wobei $6 \%$ australischen Ursprungs waren ${ }^{11}$. Es gab also generell ansprechendes Programm, geeignet oder auch sogar interessant für Kinder, aber eben nicht speziell für Kinder gemacht ${ }^{12}$.

Im Vergleich zwischen 1977/78 (368 Stunden) und 1978/79 nach der Einführung der Reglementierungen (411 Stunden) zeigt sich für das Angebot an Kindersendungen (inklusive Vorschulprogramm) ein deutlicher Anstieg. 1979/80 machten 404 Stunden Kinderprogramm $8 \%$ des Gesamtprogramms aus. Unter den neun beliebtesten Sendungen aus der Zeit zwischen 16.00 und 17.00 Uhr waren 1979/80 in Sydney fün Cklassifizierte australische Sendungen, in Melbourne vier und zwar: „Skippy" (die im Ausland erfolgreichste, auch den Kindern in Deutschland nicht unbekannte Fernsehserie mit einem Kängeruh als Star. Seit 13 Jahren ist kein Tag vergangen, an dem Skippy nicht von irgendeiner Fernsehstation der Welt ausgestrahlt worden ist. "Simon Townsend's Wonder World“ (eine unterhaltsame, aktuelle Magazinsendung für ältere Kinder mit kurzen interessanten Beiträgen), „Curiosity Show “ (für jüngere und ältere Kinder, mit naturwissenschaftlichen Themen, in der auch mit einfachen Mitteln experimentiert wird), "Shirl's Neighbourhood“ (wiederum eine heitere Magazinsendung, diesmal für jüngere Kinder, mit Shirl, einem quicklebendigen Moderator, und seinen australischen Tierpartnern, einem Känguruh und einem Oppossum - der Einfluß Walt Disney's bei der Gestaltung der Puppets ist unverkennbar - die sich auch an Aktionen in bestimmten Stadtteilen beteiligen). Aber die bei der Altersgruppe beliebtesten Sendungen waren "Wonderful World of Disney“, gefolgt von der "Muppet's Show ${ }^{\alpha}$ sowie etliche Familienserien wie "The Sullivans" oder "Happy Days" ${ }^{\text {"13 }}$. Die Situation hat sich also insgesamt doch bereits zugunsten der australischen Produktionen geändert. Geht auch der Fernsehkonsum der Kinder insgesamt zurück, so steigt die Sehbeteiligung in der C-Zeit sogar an.

Die Reglementierungen konnten nicht verhindern, daß weiterhin die meisten Kinder nicht während des Kinderprogramms vor dem Gerät sitzen, sondern in der Vorabendprogrammzeit und vor allem zwischen 19.30 und 20.30 Uhr. Da in dieser Zeit jedoch auch die Erwachsenen fernsehen, ist wenigstens eine Aufsichtsperson vorhanden, während jetzt, wenn die Kinder von der Schule nach Hause kommen - und viele Kinder unbeaufsichtigt fernsehen ${ }^{14}$ - Schonzeit ist. Insgesamt gesehen kam es also zu einem Kompromiß, der hinsichtlich der Werbung den Anstalten nicht so sehr weh tut (der Anteil der Erwachsenen zu dieser Zeit ist sehr gering, bzw. in dieser Zeit ist der Anteil 
der Kinder am Publikum am größten), aber zumindest für eine gewisse Zeit Kindern etwas speziell für sie Gedachtes bieten soll.

Und was wird zur Zeit tatsächlich angeboten? C-Programme umfassen weiterhin alte Sendungen wie z. B. "Skippy“, "Flipper" usw., die jedoch bald auslaufen werden oder die - von den meisten Stationen ausgestrahlte "Curiosity Show", die seit einigen Jahren in Produktion ist. Durch die Wiederholungsklausel wird es also interessant. Die erfolgreichste Sendung (von seiten der Industrie), die nach Einführung der CKlassifizierung entstand, ist "Shirl's Neighbourhood“, die von neun Stationen regelmäßig ausgestrahlt wird. „Simon Townsend's Wonder World“ gibt es bei sieben Stationen zu sehen.

Daneben werden viele Drehbücher beim Children's Program Committee eingereicht, die die C-Klassifikation erhalten, die aber von Fernsehanstalten - aus Kostengründen - nur selten realisiert werden. Das gesamte leicht zugängliche Material mit CKlassifikation wurde in den letzten Monaten des Jahres 1980 ausgestrahlt ${ }^{15}$.

Wozu die Regeln bislang geführt haben, waren - mit Ausnahme der Spielshow für Kinder "Matchmates", die in Melbourne im Juli 1981 anlief - eine Reihe von Magazinsendungen, die alle mehr oder weniger nach dem gleichen Muster gestrickt sind. Durch die Basisvoraussetzung der Reglementierung, daß die Fernsehanstalten fünfmal pro Woche je eine Stunde speziell für Kinder gemachtes Programm ausstrahlen müssen, ergeben sich 15 Stunden Kinderprogramm pro Woche in den Städten mit drei kommerziellen Sendern, - 780 Stunden im Jahr. Es ist demnach notwendig, neue Serien zu entwickeln, da die Produktion einzelner Sendungen viel zu kostspielig wäre.

In seinem letzten Bericht ${ }^{18}$ zieht das Children's Program Committee folgenden Schluß: Das Gesamtbild des Kinderfernsehens ist ohne $Z$ weifel besser als es vor der Einführung der C-Klassifizierung war ... Erfolge erzielte man in der Hinsicht, daß das Problem Kinderfernsehen dem betreffenden Programm-Management im Bewußtsein ist ... aber wenn nicht neue Initiativen gesetzt werden, wird das Bild statisch bleiben und so aussehen: eine halbe Stunde Magazinsendung, eine eingeführte Serie oder eine Wiederholung einer älteren australischen ... einige neue Programme werden alte ersetzen, aber alte werden weiterhin wiederholt werden. Man hatte wenigstens gehofft, daß neue Wege beschritten werden und daß die Schaffung des Bedarfs die Bereitschaft der Industrie herausgefordert hätte, um diesem Bedarf nachzukommen ... Man sah einen langen Weg vor sich, dachte aber nicht, daß so wenig geschehen würde.

Eine Änderung des gegenwärtigen Zustands wird in drei möglichen Richtungen gesehen: 1. durch Produktion nationaler Sendungen, die Kinder im gesamtaustralischen Raum interessieren, 2. durch Import ausländischer Programme, die etwas von der Welt zeigen und schließlich 3. durch Sendungen von rein lokalem Interesse. Nicht zuletzt mit dem Ziel der Schaffung "lokaler" Kindersendungen sollten Fernsehstationen fähige Mitarbeiter heranziehen, die eine Verbindung zwischen den einzelnen Fernsehstationen und dem Publikum herstellen, gegenseitiges Wissen ermöglichen, Kontakte schaffen können.

Der Bericht impliziert die Schlußfolgerung, daß die geforderten Bedingungen wahrscheinlich ungenügend waren und man sie wird erhöhen müssen. Gegen diese Forderungen stellt sich freilich die Industrie, war sie doch schon mit den ursprünglichen Anforderungen nicht einverstanden. F.A.C.T.S. kritisiert die ProgrammstandardsRichtlinien als vage und subjektiv, nicht präzise, schwer umsetzbar usw. Sie sieht ein 


\section{Schaubild:}

Das Kinderfernseh-Angebot der kommerziellen Fernsehstationen in Melbourne zwischen 16.00 und 18.00 Uhr.

\section{HSV 7}

GTV 9

ATV 10

\section{MONDAY}

4.00 Flipper

4.30 Shirl's Neighbourhood

5.00 Wheel of Fortune

5.30 Get Smart
4.00 Skippy

4.30 Matchmates

5.00 Gomer Pyle U.S.M.C. $\quad 4.00$ Wonder World

5.30 Family Feud

5.55 News
5.00 Batman

5.30 The Brady Bunch

\section{TUESDAY}

4.00 Flipper

4.30 Shirl's Neighbourhood

5.00 Wheel of Fortune

5.30 Get Smart
4.00 Skippy

4.30 Matchmates

5.00 Gomer Pyle U.S.M.C.

5.30 Family Feud

5.55 News
4.00 Wonder World

5.00 Batman

5.30 The Brady Bunch

\section{WEDNESDAY}

4.00 Flipper

4.30 Shirl's Neighbourhood

5.00 Wheel of Fortune

5.30 Get Smart
4.00 Skippy

4.30 Matchmates

5.00 Gomer Pyle U.S.M.C. $\quad 4.00$ Wonder World

5.30 Family Feud

5.55 News
5.00 Batman

5.30 The Brady Bunch

\section{THURSDAY}

4.00 Flipper

4.30 Shirl's Neighbourhood

5.00 Wheel of Fortune

5.30 Get Smart
4.00 Skippy

4.30 Matchmates

5.00 Gomer Pyle U.S.M.C. $\quad 4.00$ Wonder World

5.30 Family Feud

5.55 News
5.00 Batman

5.30 The Brady Bunch

\section{FRIDAY}

4.00 Flipper

4.30 Shirl's Neighbourhood

5.00 Wheel of Fortune

5.30 Get Smart
4.00 The Curiosity Show

4.30 Matchmates

5.00 Gomer Pyle U.S.M.C.

5.30 Family Feud

5.55 News
4.00 Wonder World

5.00 Batman

5.30 The Brady Bunch

Das hier angeführte Beispiel einer Woche (14. bis 18. September 1981) der kommerziellen Stationen in Melbourne kann als Muster für die "Streuung“ des Angebots der 50 kommerziellen Stationen, die ja wiederum zu Networks zusammengeschlossen sind, stehen. Gibt es bei einzelnen Stationen auch eine andere Zusammenstellung und andere Sendungen, so ändert sich doch am Prinzip nichts. 
Problem in der Tatsache, daß eine kleine Gruppe von ausgewählten Personen das Angebot sichtet (die Kommission trifft sich jeden Monat und bewertet eingereichte Sendungen, Manuskripte usw.) und Sendungen nach eigenen Regeln approbiert ${ }^{17}$.

Auch in den Printmedien wurde bei der Einführung die Reglementierung heftig kritisiert ${ }^{18}$. Man verwies darauf, daß z. B. bei der Sichtung der Programmvorschläge etwa die Hälfte der eingereichten Sendungen nicht in die C-Gruppe aufgenommen wurde und wirft der Kommission vor, Regeln unausgegoren aufgestellt zu haben. Der Sprecher der F.A.C.T.S. wird zitiert, der davon sprach, daß zum ersten $\mathrm{Mal}$ in der Geschichte des Rundfunks in Australien eine Regierungsstelle volle Kontrolle über einen Teil des Programms übernommen hat, dem Zuseher diktiert, was er sehen darf. Vergleiche mit Zensur einer Zeitungsseite werden angestellt. Komitee-Mitglieder müssen sich Eierköpfe nennen lassen. Gemüter erhitzen sich weiterhin an Entscheidungen bezüglich der Klassifizierung - bzw. Nicht-Klassifizierung - von Sendungen nicht selten wird Zensur als undemokratisches Beispiel ins Spiel gebracht, man glaubt die Gefahr zu sehen, daß das ABT die Kontrolle auch auf andere Programmsparten ausdehnen möchte.

In der Bevölkerung jedoch schätzt man die Reglementierung, wie eine Befragung Ende 1979 zeigt. 87 Prozent befürworten diese Zeitspanne mit Sendungen speziell für schulpflichtige Kinder - gedacht für deren Unterhaltung.

\section{Die Australian Cbildren's Television Foundation ${ }^{19}$}

$\mathrm{Da}$ in dieser Situation Regeln und Forderungen allein nicht erreichen konnten, was verlangt wird, erscheint es unrealistisch, von der Industrie zu erwarten, daß ausschließlich sie die Bürde der Entwicklung neuer Programmformen trägt. Man sieht die Problematik und die Schwierigkeiten, Programm für ein derartig divergierendes Publikum wie Kinder zwischen 6 und 13 Jahren zu machen - es erfordert eine spezielle Schulung und enthält Risiken. Will man tatsächlich eine Verbesserung erreichen, heißt es, Regierungen, Networks und andere Geldgeber zu vereinen und kooperativ ans Werk zu gehen. Genau diesen Zweck verfolgt die Australien Children's Television Foundation.

Die Initiative zu dieser unabhängigen Körperschaft mit nationaler Identität und ohne kommerzielle Interessen geht (abgesehen von Vorschlägen für eine unabhängige Produktionseinheit für Film und Fernsehen für Kinder, die in der Vergangenheit schon mehrfach gefordert worden war) auf den Minister for the Arts in Victoria zurück, der von anderen Regierungsstellen in Australien unterstützt wurde. Die Stiftung sieht ihre Funktion in der Ermutigung zur Entwicklung, Produktion, Ubertragung von Qualitäts-Kinderfernsehen, in der Schulung (einschließlich spezieller Trainings für Autoren, Regisseure, Produzenten), in der Suche nach möglichen Sponsoren zur Unterstützung von Forschung in allen für Kinder und Fernsehen relevanten Bereichen, in der Propagierung von Medienerziehung, in der Sammlung und Verarbeitung von Information zum Fernsehen für Kinder, im Ausfindigmachen von ausländischen Qualitätssendungen und deren Einfuhr nach Australien sowie im Marketing und der Anpassung von Material für den Verkauf ins Ausland.

Ein halbes Jahr lang arbeitete eine kleine Gruppe von Personen an Arbeitsgrundlagen für die Stiftung und ebnete den Weg für die Unterstützung bei Regierungsstellen nun durch konkrete finanzielle Beiträge. Man braucht im ersten Jahr $500000 \$$ (das sind etwa 1,5 Mill. DM), um die Arbeit aufnehmen zu können, und ähnliche Summen 
für die folgenden zwei Jahre. Dann beabsichtigt man, sich selbst finanzieren zu können.

Scheint diese Initiative für die Intensivierung der Forschung und die Verbreiterung der Angebotsvielfalt durch die Steigerung der Attraktivität auch der Arbeitsplätze in diesem Bereich auch durchaus vernünftig und unterstützenswert, so fürchten doch bereits jetzt bestehende kleinere Organisationen mit teilweise ähnlichen Zielen um ihre Existenzberechtigung und -grundlage ${ }^{20}$.

Die Industrie verhält sich mit Stellungnahmen noch abwartend, genauso manche (Staats-)Regierungsstellen, die noch nicht bereit sind, diese auf nationaler Ebene arbeitende Initiative zu unterstützen. Die Schwierigkeiten sind also noch lange nicht alle beseitigt. Als Hindernis stellen sich die bereits eingefahrenen Bahnen aus der Arbeit mit den inzwischen seit zwei Jahren bestehenden Regeln dar. Man konnte auch mit nicht allzu viel Aufwand den Anforderungen genügen. Dennoch ist der Start der Children's Television Foundation ein großer Erfolg und eine große Chance für das Kinderfernsehen - nicht nur in Australien.

Diese Stiftung wird dabei keineswegs das Children's Program Committee ersetzen. Sie baut vielmehr auf dessen verstärkter Autorität auf. Das CPC hat u. a. weiterhin die Aufgabe, eingereichtes Material für die Ausstrahlung zwischen 16.00 und 17.00 Uhr hin zu klassifizieren und das Problem der nachlassenden Qualität bei Sendungen, die schon länger in Produktion sind, zu lösen - eventuell verschärfte Regeln einzuführen. Jedenfalls werden viele Faktoren zusammenarbeiten müssen, um das übergeordnete Ziel auch tatsächlich zu erreichen. Die Foundation hat nun drei Jahre Zeit und die ersten Produktionsaufträge wurden auch schon vergeben ${ }^{21}$.

Anmerkungen:

1 Vgl. dazu u. a.: Australian Broadcasting Tribunal: Television and Children. Australia 1977/78. Australian Broadcasting Tribunal Reserach Report based on surveys conducted in Melbourne, Sydney, Adelaide and Brisbane in 1977 (Sydney, 1978); ferner Peter Cook: How Much Television do Australian Children Watch? A Survey of Research for the Active Eye Project. Active Eye Project Working Paper 5. Murdoch University, Mount Lawley College (Perth, 1981).

2 Der Bericht enthält Vorschläge für die Neuformulierung von Programmstandards, für Fernsehwerbung in der Zeit des Kinderfernsehens, für Fernsehspielproduktionen für Kinder, die nun in der Offentlichkeit diskutiert werden sollen, bevor sie in Kraft treten können.

CPC-Children's Program Committee: Third Progress Report, (Sydney) May 1982.

3 Die Australian Broadcasting Commission (A.B.C.) wie auch die Public Television und Special Broadcasting Services sowie Ethnic Television bleiben von den Ausführungen ausgenommen, da für sie nicht die Reglementierungen des Australian Broadcasting Tribunal gelten. Die A.B.C. nimmt zusätzlich eine Sonderstellung ein, da sie jüngere Kinder mit 90 Minuten Vorschulprogramm ("Sesame Street ${ }^{\alpha}$ und „Playschool ${ }^{\text {) }}$ ) und ältere Kinder mit weiteren 90 Minuten abwechslungsreichem Programm australischen (zu etwa 50\%), britischen, neuseeländischen, amerikanischen etc. Ursprungs sehr gut versorgt.

- Vgl. dazu Patricia Edgar: Improving view of four millions. In: The Age, Green Guide, February 26, 1981, p. 7.

Anmerkung: Zirka $6 \%$ des Budgets der kommerziellen Fernsehstationen wird für lokale Kindersendungen ausgegeben. 
Das Kinderprogramm-Angebot der A.B.C. (Melbourne) zwischen 15.00 und 18.00 Uhr in der Woche vom 14. bis 18. September 1981:

\begin{tabular}{|c|c|c|}
\hline Monday & Tuesday & Wednesday \\
\hline $\begin{array}{l}\text { 3.05 Sesame Street } \\
\text { 4.00 Playschool } \\
\text { 4.30 Mr Squiggle } \\
\text { 5.00 Grange Hill } \\
\text { 5.30 Park Ranger } \\
\text { 5.58 News Headlines }\end{array}$ & $\begin{array}{l}\text { 3.00 Sesame Street } \\
\text { 4.00 Play School } \\
\text { 4.30 Earth Watch } \\
\text { 5.00 The Nargun and the } \\
\text { Stars } \\
\text { 5.58 News }\end{array}$ & $\begin{array}{l}\text { 3.00 Sesame Street } \\
\text { 4.00 Play School } \\
\text { 4.30 The Basil Brush Show } \\
\text { 5.00 Children of the Fire } \\
\text { Montain } \\
\text { 5.30 The Swish of the } \\
\text { Curtain } \\
\text { 5.58 News }\end{array}$ \\
\hline
\end{tabular}

\begin{tabular}{l|l}
\multicolumn{1}{c|}{ Thursday } & \multicolumn{1}{|c}{ Friday } \\
\hline 3.05 Sesame Street & 3.00 Sesame Street \\
4.00 Play School & 4.00 Play School \\
4.30 Spectrum & 4.30 Famous Five \\
5.00 The Flying Kiwi & 5.00 Matt and Jenny \\
5.30 Our John Willie & 5.30 The Enchanted Castle \\
5.58 News & 5.58 News \\
\hline
\end{tabular}

1975/76 waren es 4,9 Millionen Dollar, die für die Produktion von Kinder-Fernsehen aufgewendet wurden.

Vgl. dazu David Morgan: Production, Marketing and Distribution: Children's Television. In: Report of the Australian Children's Film and Television Seminar - Program for Tomorrow. Canberra, October 12-16, 1977. Open Program. Australian Film and Television School, 1977.

5 Vgl. dazu Gespräch mit Jenny Hooks, Produzent der erfolgreichen Kindersendung „Shirl's Neighbourhood ${ }^{\alpha}$, Channel 7 Network, Melbourne, am 3. August 1981.

- Vgl. dazu: Senate Standing Committee on Education and the Arts: Inquiry into The Impact of Television on the Development and Learning Behaviour of Children. Australian Government Publishing Service, Canberra, 1978 sowie: Children and Television Revisited. A Review of $t$ he Report on the Impact of Television on the Development and Learning Behaviour of Children (1981).

Weiter: Children's Program Committee: Report on the first year (November 1978 November 1979). In: Australian Broadcasting Tribunal: Annual Report 1979-80. Australian Government Publishing Service, Canberra, 1980, p. 189-198.

Patricia Edgar: Children's television - the past, the present, the future Second Annual John Grierson Lecture, State Film Centre. November 15, 1978, (Melbourne, 1978).

Frank de Chiera: The "C"Story. In: TV Probe. Children Television: The Great Debate (1980), p. 5-12.

7 Vgl. dazu u. a.: Children's Program Committee: Progress Report. o. J. (1981) Manuskript. Zum besseren Verständnis der C-Klassifizierung sei kurz angemerkt, daß im australichen Fernsehen hinsichtlich der zeitlich möglichen Ausstrahlung eine "Zensur" stattfindet. So müssen Sendungen, die jederzeit ausgestrahlt werden können eine G (general audience) Klassifizierung haben. PGR (parential guidance recommended)-klassifiziertes Material darf nicht $z$ wischen 6.00 und 8.30 sowie 16.00 und 19.30 Uhr wochentags und 6.00 bis $19.30 \mathrm{Uhr}$ Samstag/Sonntag gesendet werden. Programm für Adults only (AO) darf zwischen 12.00 und 15.00 Uhr und ab 20.30 Uhr wochentags zur Ausstrahlung gelangen, an Wochenenden ab 20.30 Uhr. Weiter gibt es Sendungen, die überhaupt nicht im 
Fernsehen gezeigt werden dürfen. Insgesamt gesehen soll die Zeit zwischen 6.00 und 8.30 sowie 16.00 und 19.30 Uhr für Kinder ,sicher" sein.

Die C-Gruppe ist demnach eine Präzisierung der zur generellen Ausstrahlung zugelassenen Gruppe mit dem Ziel, Programmverantwortliche zu ermutigen, neue Programme speziell für Kinder zu entwickeln, um den speziellen Bedürfnissen der Kinder Rechnung zu tragen. Diese Sendungen sollten in einer Art und Weise gestaltet und präsentiert werden, daß sie leicht verständlich sind und Kinder ansprechen, was aber keineswegs heißt, daß die Sendungen in erster Linie erzieherisch oder bildend sein sollen - wie in Europa, wo Kinderprogramm in erster Linie der geistigen Entwidklung dienen soll. Im Gegenteil, gutes Kinderprogramm muß zu allererst unterhaltend sein. Daher wird ein ${ }^{\circ} \mathrm{C}^{4}$ auch nur solchen Programmen zuerkannt, die speziell auf die Altersgruppe zwischen 6 und 13 Jahren zugeschnitten sind. Die Empfehlungen für die Produzenten enthalten dazu den Hinweis, eine Vielfalt von Sendtypen zu entwidkeln und sechs Stunden Fernsehspiel für Kinder pro Jahr zu zeigen. Wiederholungen sollten nur ein Mal in zwei Jahren gestattet sein.

V Vgl. dazu: Children's Program Committee. Australian Broadcasting Tribunal: The Developing Child. Australian Broadcasting Tribunal, Sydney, 1980.

- Vgl. dazu Ian Fairweather: Kids' TV. 433 Things to Do in a Rainy Studio. NBN Limited/Southern Television Corporation Limited, (o. O.), 1981.

10 Vgl. dazu Patricia Edgar: Two Approaches to the Regulation of Children's Television. Manus. (1981), p. 2.

11 Vgl. dazu: Senate Standing Committee on Education and the Arts: Inquiry into The Impact ... a. a. O., p. $71 \mathrm{ff}$.

12 Anmerkung: Im "Active Eye Project" wird von "polisemic programmes" gesprochen, d. h. man sucht Programmformen, Gestaltungsmittel und Produktionswege für Sendungen, die auf mehreren Ebenen Informationen enthalten. Kinder sollen davon ebenso angesprochen werden wie Erwachsene und Kinder sollen nicht für eine Stunde in ein "kindgemäßes Getto $^{*}$ abgeschoben werden. Man will verhindern, daß durch Reglementierung eine Barriere zwischen den beiden Welten verstärkt wird.

Vgl. dazu Bob Hodge: The Regulation of Children's TV in Australia. Active Eyc Project Working Paper 1. Murdoch University, Mount Lawley College (Perth, 1981).

10 Vgl. dazu: Australian Broadcasting Tribunal: Annual Report 1979-80. a. a. O.

14 Vgl. dazu: Australian Broadcasting Tribunal: Television and Children. Adult Viewing in Children's Time. Sydney - 1979. Australian Broadcasting Tribunal Research Report. Based on a Survey Conducted in Syndey June 1979.

16 Vgl. dazu: Children's Program Committee: Progress Report ... a. a. O.

Anmerkung: Der ABT befaßt sich zur Zeit mit der Empfehlung des CPC, von den kommerziellen Fernsehstationen die Produktion von sechs Stunden Fernsehspiel für Kinder im ersten Jahr und zehn ab dem folgenden zu verlangen. Die $A B C$ ist bislang die einzige Fernsehanstalt in Australien, die in dieser Richtung aktiv ist. Der Sprecher der F.A.C.T.S. argumentiert allerdings mit den Produktionskosten gegen solche Forderungen. (Kosten eine halbe Stunde Magazinsendung derzeit ca. AUS $\$ 4.000$ bis 5.000 ,-, wären für eine halbe Stunde Fernsehspiel $\$ 35.000$, - und mehr zu veranschlagen - ebensoviel wie für das Erwachsenenprogramm, z. B. die erfolgreiche Vorabendserie „Prisoner“.)

10 Vgl. dazu: Children's Programm Committee: Progress Report ....a. a. O.

${ }^{17}$ Vgl. dazu: Children's Television. In: F.A.C.T.S. 78/79 Report, p. 19-22.

18 Vgl. dazu diverse Zeitungsberichte aus 1979.

10 Vgl. dazu: The Australian Children's Television Foundation. Broschüre o. O., o. J. (1981), sowie Gespräche mit Dr. Patricia Edgar, Senior Lecturer, La Trobe University, Media Centre; Präsidentin des Children's Program Committee des ABT und Verantwortliche für die Entwicklung der Arbeitsgrundlagen der Foundation.

20 Vgl. dazu: Jahreshauptversammlung des Victorian Council for Children's Film and Television am 25. Mai 1981 in Melbourne.

21 Vgl. dazu: Gespräch mit der Präsidentin der Children's Television Foundation, Dr. Patricia Edgar anläßlich des Prix Jeunesse im Juni 1982 in München. 


\section{SUMMARY}

One can assume that commercial television station licencees are not very keen on producing children's programmes or putting expensive imported ones on air. Taking Australia as an example, this article looks at the effects of the introduction of regulations and the necessary classification procedure of programmes for 6 to 13 year olds to be broadcast between 4 and $5 \mathrm{pm}$.

Censurshiplike means applied according to recommendations of the Children's Program Committee created the need for programme material which could only be fulfilled by producing cheaper magazine programmes. More strictly applied requirements finally lead to the establishment of the Children's Television Foundation, which during the first three years is supported by governments, but is working as an independent foundation. Its aim is to have developed and produced quality children's programmes. It also will be in charge of the distribution of the programmes. Another task will be the special training of production teams and also research and promotion of media education.

\section{RÉSUME}

Des règlements légaux pour stations de télévisions commerciales sont apparemment l'unique possibilité pour offrir des émissions spéciales pour enfants. Prenant l'Australie pour exemple, cet article regarde les effets de l'introduction des règlements et la procédure de classification des programmes pour enfants de 6 à 13 ans qui doivent être transmis entre 16.00 et 17.00 heures. Les directives du Children's Program Committee, semblant à une censure, ont créé un besoin qui n'a pu être suivi par les stations de télévision que par la production des programmes de feuilletons bon marché. Les exigences élevées conduisaient enfin à la création de la Children's Television Foundation, une fondation indépendante mais supportée pendant les premiers trois ans par le Gouvernement, ayant comme but le développement et la production des émissions de qualité pour les enfants. La distribution des programmes est faite par la même fondation. Ses autres buts sont la formation de producteurs de programmes pour enfants, la recherche et ha promotion de l'éducation aux média.

\section{RESUMEN}

Se puede suponer que los responsables de estaciones comerciales de televisión no estan muy entusiasmados en la producción de programas para niños o en la difusión de costosos programas importados. - Tomando el ejemplo de Australia, este artículo considera los efectos que tuvo la introducción de reglamentaciones y el necesario procedimiento de clasificación de programas que serán transmitidos entre las cuatro y cinco de la tarde para niños de seis a trece años.

La aplicación de medidas que se asemejan a una censura y en acuerdo a las recomendaciones del Comité de Programas para Niños, creó la necesidad de nuevos materiales de programa los cuales podrían ser logrados sólo a través de la producción de programas de bajo costo del tipo „magazine“. Las exigencias cada vez más estrictas condujeron finalmente a la creación de una Fundación de Televisión Infantil, la cual durante los tres primeros años es apoyada por el Gobierno, pero que trabaja de manera independiente. Su propósito es desarrollar y producir programas de calidad para los niños, ocupándose también de la distribución de estos programas.

Otra tarea de esta Fundación será la capacitación especial para equipos humanos de producción y tambien la investigación y promoción de la educación de los „media“. 